\title{
INFLUÊNCIA DO MEIO REACIONAL NO COMPORTAMENTO FOTOQUÍMICO DO INSETICIDA PARATION ETÍLICO
}

\author{
Francismário Ferreira Santos e Maria Olímpia Oliveira Rezende
}

Instituto de Química de São Carlos, Universidade de São Paulo, 13560-970 São Carlos - SP

Recebido em 1/3/01; aceito em 9/5/01

\begin{abstract}
INFLUENCE OF REACTION MEAN IN THE BEHAVIOR PHOTOCHEMICAL OF INSECTICIDE ETHYL PARATHION. The photodegradation of parathion in natural and dezionised waters was studied under irradiation at two different wavelengths: 280 $\mathrm{nm}$ and $313 \mathrm{~nm}$. The influence of humic acids was evaluated. The results demonstrated that the degradation occurred only due to photochemical processes. The chemical hydrolysis and biological processes can be neglected in this case. The addition of humic acids did not increase the photodegradation rate in either water samples (natural or dezionised). In alkaline solutions the photodegradation rate was higher in dezionised water when compared to natural waters. The kinetic degradation in all experiments obeyed a first order reaction pattern.
\end{abstract}

Keywords: humic acids; photodegradation; parathion.

\section{INTRODUÇÃo}

Um grande número de substâncias tóxicas orgânicas e inorgânicas são introduzidas no ambiente como descarte. Dentre essas, os pesticidas foram e continuam sendo as principais substâncias empregadas na eliminação de qualquer forma de vida que venha a prejudicar a produtividade agrícola das culturas. Devido ao uso extensivo dos pesticidas, muitas vezes indiscriminado, sua presença em solos e ambientes aquáticos tem aumentado. Estima-se que 2,5 milhões de toneladas de pesticidas foram aplicados em solos e folhagens em $1996^{1}$

A persistência e alta toxicidade de alguns pesticidas têm motivado muitas pesquisas com o objetivo de encontrar métodos de remoção de poluentes orgânicos do ambiente ${ }^{2,3}$. Atualmente, dentre os processos de descontaminação, os processos fotoquímicos, conhecidos como processos de oxidação avançados (POA), são considerados muito promissores devido à possibilidade de mineralização total ou parcial de poluentes orgânicos ${ }^{4-8}$.

A matéria orgânica decomposta, especialmente as substâncias húmicas $(\mathrm{SH})$, é constituída de macromoléculas complexas, proveniente da decomposição de plantas e resíduos de animais. Operacionalmente, as $\mathrm{SH}$ podem ser classificadas em três principais frações: ácidos húmicos (AH), ácidos fúlvicos (AF) e humina. Devido à presença de vários grupos funcionais, carboxilas, hidroxilas alcoólicas e fenólicas, carbonilas, ésteres etc., as SH podem interagir com materiais orgânicos e inorgânicos por meio de processos físicos e químicos e, assim, afetar o destino final destes no ambiente. No ambiente, as SH podem absorver a radiação ultravioleta (UV) da luz solar e gerar espécies reativas como radicais hidroxilas $(\mathrm{OH})$, elétron solvatado $\left(\mathrm{e}_{\mathrm{aq}}{ }^{-}\right)$, oxigênio singlete molecular $\left({ }^{1} \mathrm{O}_{2}\right)$ etc. ${ }^{5,9-11}$. Essas espécies podem fotoinduzir a tranformação de compostos que não absorvem diretamente a radiação UV ${ }^{12}$ através de transferência de energia ${ }^{13,14}$. O emprego da radiação UV com outras substâncias como óxido de titânio $\left(\mathrm{UV} / \mathrm{TiO}_{2}\right)^{15,16}$ e peróxido de hidrogênio (UV/ $\mathrm{H}_{2} \mathrm{O}_{2}$ ) ${ }^{17}$ é muito estudado, no entanto, poucos trabalhos apresentam resultados sobre a degradação induzida de pesticidas associados a AH. Assim, o objetivo deste trabalho foi avaliar o processo fotoquímico como uma alternativa de descontaminação de poluentes em águas.
O inseticida e acaricida paration etílico é um pesticida organofosforado de ação fulminante, utilizado em uma variedade de culturas, como inibidor da enzima acetilcolinesterase.

No presente trabalho é apresentado o comportamento fotoquímico do pesticida paration em águas, na presença e ausência de $\mathrm{AH}$. $\mathrm{O} \mathrm{pH}$ do meio e o comprimento de onda de irradiação também foram avaliados. O paration foi utilizado devido a ser um dos pesticidas mais tóxicos da classe dos organofosforados e devido à possibilidade de contaminar o ambiente, em particular, solos e águas.

\section{MATERIAIS E MÉTODOS}

\section{Materiais e reagentes}

Foi utilizado o pesticida paration etílico $\left(\mathrm{C}_{10} \mathrm{H}_{7} \mathrm{O}_{2} \mathrm{PS}\right)$ com pureza de $96 \%$. Os AH utilizados nos experimentos foram extraídos de turfa, de acordo com o método padrão baseado em extrações ácidobase seguidas de purificação com resinas catiônicas e aniônicas ${ }^{18}$. As principais características dos AH utilizados são apresentadas na Tabela 1.

Tabela 1. Características dos AH utilizados ${ }^{19}$

\begin{tabular}{lr}
\hline Cinzas \% & 2,80 \\
$\mathrm{C} \%$ & 53,72 \\
$\mathrm{~N} \%$ & 1,97 \\
$\mathrm{H} \%$ & 3,87 \\
$\mathrm{O}^{\mathrm{a}} \%$ & 37,64 \\
Acidez total, $\mathrm{mmol} / \mathrm{g}$ & 5,11 \\
Acidez carboxílica, mmol/g & 3,18 \\
Acidez fenólica ${ }^{\mathrm{a}}, \mathrm{mmol} / \mathrm{g}$ & 1,93 \\
\hline
\end{tabular}

${ }^{a}$ Valores obtidos por diferença para $100 \%$.

\section{Fonte de irradiação e reator fotoquímico}

Todos os experimentos fotoquímicos foram realizados em um reator cilíndrico de $400 \mathrm{~mL}$ de capacidade e equipado com uma jaqueta de quartzo, Figura 1. Como fonte de luz UV foi utilizada uma lâmpada de mercúrio de média pressão (ACE glass Inc., NJ, USA), 
450 Watts, 5" de comprimento, com filtro de quartzo para comprimento de onda maior que $280 \mathrm{~nm}$.

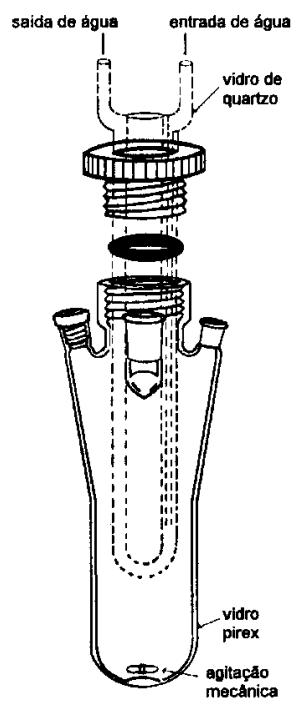

Figura 1. Esquema representativo do reator fotoquímico.

\section{Procedimento fotoquímico}

As soluções irradiadas foram preparadas com a adição de $10 \mathrm{mg} / \mathrm{L}$ de paration em uma solução tampão fosfato $(0,025 \mathrm{~mol} / \mathrm{L}) \mathrm{pH} 5,50 \mathrm{e}$ 7,25 , na ausência e presença de $\mathrm{AH}(10 \mathrm{mg} / \mathrm{L})$. As soluções foram preparadas utilizando-se água desionizada e água do Reservatório de Captação Superficial Anhumas, localizado em Américo Brasiliense, SP. As soluções foram mantidas sob agitação magnética e refrigeração durante todo o experimento. Os experimentos fotoquímicos na presença e ausência de $\mathrm{AH}$ foram realizados por um período de $30 \mathrm{~min}$ retirando-se alíquotas de $10 \mathrm{~mL}$ nos tempos de 2, 5, 10, 15, 20, 25 e $30 \mathrm{~min}$. Foi realizado, também, um experimento fotoquímico com água desionizada, na ausência de $\mathrm{AH}$, empregando-se um filtro de luz (vidro pirex) entre a lâmpada e a solução para obter $\lambda>313 \mathrm{~nm}$. Este experimento foi realizado retirando-se alíquotas de $10 \mathrm{~mL}$ em intervalos de 1 hora por um período de 8 horas de irradiação. Após as irradiações, as amostras foram acondicionadas no escuro, a $5{ }^{\circ} \mathrm{C}$, por um período de 30 dias. Todas as soluções foram extraídas três vezes com acetato de etila, com porções de $10 \mathrm{~mL}$. Em seguida, foram rotaevaporadas e ressuspendidas com $10 \mathrm{~mL}$ de metanol.

\section{Metodologia analítica}

A determinação do paration foi realizada por cromatografia líquida, utilizando um cromatógrafo Shimadzu modelo SPD-6A, equipado com detector UV-Vis LC-9A (HPLC-UV). A detecção foi realizada em $280 \mathrm{~nm}$ (comprimento de onda de absorção do paration). Foi utilizada uma coluna $\mathrm{C}_{8}$ (Shim-pack) para separação do paration e seus fotoprodutos. Como eluente foi utilizada a mistura metanol/ água $(70: 30 \mathrm{v} / \mathrm{v})$ e vazão de $1 \mathrm{~mL} / \mathrm{min}$.

A confirmação dos possíveis fotoprodutos formados durante os experimentos foi feita utilizando um cromatógrafo a gás HP 5890 acoplado a um detector seletivo de massas (GC-MS).

\section{RESULTADOS E DISCUSSÃO}

A influência dos AH na fotodegradação do paration foi estudada em amostras de águas naturais e desionizada. Apesar do paration ser o mais resistente à hidrólise química entre os organofosforados, particularmente em valores de $\mathrm{pH}$ na região neutra e ácida ${ }^{20}$, duplicatas de alíquotas de $50 \mathrm{~mL}$ de todas as amostras em estudo foram acondicionadas no escuro, em acompanhamento aos experimentos fotoquímicos, de forma a avaliarmos qual a contribuição da hidrólise na degradação do paration quando da presença de AH em solução. Os resultados das análises das amostras demonstraram que o paration foi recuperado totalmente. Isto nos leva a dizer que a hidrólise química pode ser negligenciada nas condições dos experimentos. A recuperação total do paration nos experimentos mostra, também, que não ocorreu degradação por meio da ação biológica, mesmo após um período de 30 dias, no escuro, e nem por adsorção pelos $\mathrm{AH}$. Assim, podemos concluir que a degradação do paration foi mediada pela ação da radiação UV.

\section{Influência do meio na degradação do paration em água desionizada}

O efeito dos AH na fotodegradação do paration em solução aquosa, preparada com água desionizada, pode ser verificado na Figura 2, em pH 5,5.

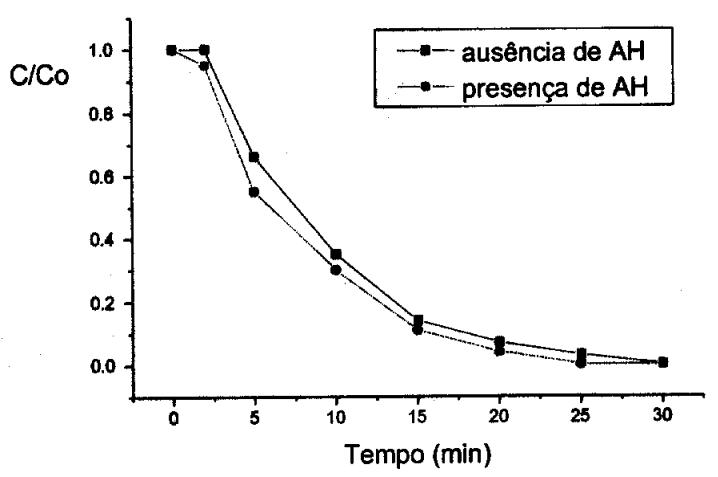

Figura 2. Concentração relativa de paration em água desionizada, na ausência e presença de AH, pH 5,5, com irradiação em $\lambda>280 \mathrm{~nm}$.

Como pode ser visto, a degradação total do paration ocorreu em 25 min e 30 min na presença e ausência de AH, respectivamente. Estes resultados mostram que a presença de $\mathrm{AH}$ pouco influenciou na velocidade de fotodegradação do paration. As constantes de velocidade de fotodegradação, com e sem $\mathrm{AH}$, foram determinadas em termos do logaritmo da concentração de paration remanescente em solução, em função do tempo de irradiação. Considerou-se uma reação de cinética de $1^{\text {a }}$ ordem, sendo $\mathrm{k}=0,161 \mathrm{~min}^{-1}$ e $\mathrm{k}=0,138 \mathrm{~min}^{-1}$ os valores determinados na presença e ausência de $\mathrm{AH}$, respectivamente.

O paration também foi irradiado em $\mathrm{pH}$ 7,25 para avaliar a influência do $\mathrm{pH}$ do meio. $\mathrm{Na}$ presença de $\mathrm{AH}$, a degradação total do paration ocorreu em 15 min. Na ausência de $\mathrm{AH}$, a degradação total ocorreu em 20 min. Da mesma forma, houve pouca influência do AH na velocidade de fotodegradação, sendo $\mathrm{k}=0,253 \mathrm{~min}^{-1} \mathrm{e}$ $\mathrm{k}=0,223 \mathrm{~min}^{-1}$ as constantes de velocidade na presença e ausência de AH, respectivamente, Figura 3.

No entanto, ao compararmos os valores das constantes de velocidade de fotodegradação em pH $5,5\left(\mathrm{k}=0,138 \mathrm{~min}^{-1}\right)$ e 7,25 $\left(\mathrm{k}=0,223 \mathrm{~min}^{-1}\right)$ na ausência de $\mathrm{AH}$, podemos notar que o aumento do $\mathrm{pH}$ aumentou a velocidade de fotodegradação do paration, Figura 4. O tempo de meia-vida na ausência e presença de AH em pH 5,5 $\left(\mathrm{t}_{1 / 2} \cong 6\right.$ e $8 \mathrm{~min}$, respectivamente) é maior quando comparado ao sistema em pH 7,25 ( $\mathrm{t}_{1 / 2} \cong 5$ e $6 \mathrm{~min}$, respectivamente). No entanto, na ausência de $\mathrm{AH}$, o $\mathrm{t}_{1 / 2}$ do paration em $\mathrm{pH} 7,25\left(\mathrm{t}_{1 / 2} \cong 5 \mathrm{~min}\right)$ é 


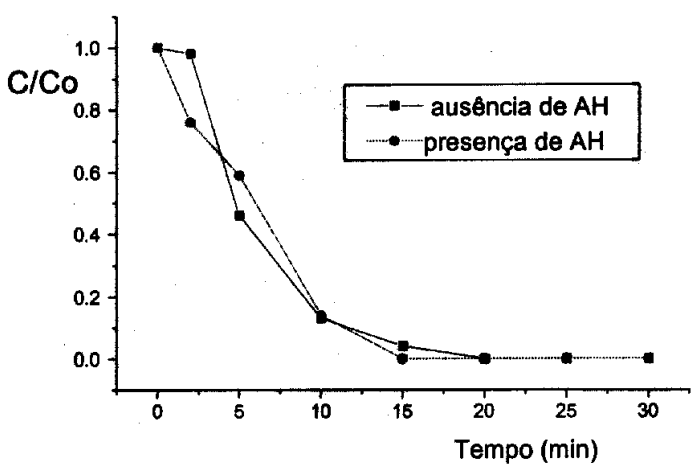

Figura 3. Concentração relativa de paration em água desionizada, na ausência e presença de $\mathrm{AH}, \mathrm{pH}$ 7,25, com irradiação em $\lambda>280 \mathrm{~nm}$.

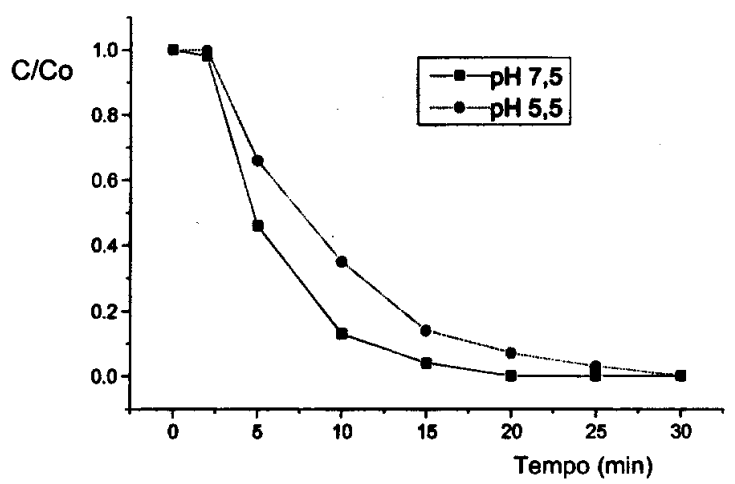

Figura 4. Concentração relativa de paration em água desionizada, na ausência $\mathrm{AH}, \mathrm{pH}$ 5,5 e 7,25, com irradiação em $\lambda>280 \mathrm{~nm}$.

menor do que o verificado em $\mathrm{pH} 5,5\left(\mathrm{t}_{1 / 2} \cong 8 \mathrm{~min}\right)$. Assim, pode-se dizer que o $\mathrm{pH}$ do meio teve maior influência na fotodegradação do paration do que a presença do $\mathrm{AH}$ no meio reacional.

Tal conclusão pode ser verificada mais claramente por meio do planejamento fatorial envolvendo as variáveis $\mathrm{AH}$ e $\mathrm{pH}$ em estudo ${ }^{21}$. Além disso, a análise fatorial permite verificar a existência de interações entre as variáveis. A Tabela 2 apresenta o planejamento fatorial para calcular o efeito das variáveis $\mathrm{AH}$ e $\mathrm{pH}$ em função dos resultados fotoquímicos.

Tabela 2. Resultados de um planejamento fatorial $2^{2}$ para estudar o efeito do $\mathrm{pH}$ e do $\mathrm{AH}$ na fotodegradação

\begin{tabular}{|c|c|c|c|}
\hline \multicolumn{2}{|c|}{$\begin{array}{c}\text { Variável } \\
\mathrm{pH} \\
\mathrm{AH}\end{array}$} & $\begin{array}{c}\text { Nível (-) } \\
5,5 \\
\text { Não }\end{array}$ & $\begin{array}{c}\text { Nível (+) } \\
7,25 \\
\text { Sim }\end{array}$ \\
\hline pH & $\mathbf{A H}$ & $\mathbf{p H} \times \mathbf{A H}$ & $\begin{array}{l}\text { Resposta } \\
\left(\mathbf{k}, \text { min }^{-1}\right)\end{array}$ \\
\hline- & - & + & 0,138 \\
\hline+ & - & - & 0,223 \\
\hline- & + & - & 0,161 \\
\hline+ & + & + & 0,253 \\
\hline
\end{tabular}

A partir dos valores da Tabela 2, o efeito dos AH e pH foi calculado como segue:

pH: $(-0,138+0,223-0,162+0,253) / 2=+0,088$

AH: $(-0,138-0,223+0,162+0,253) / 2=+0,026$

pH x AH: $(0,138-0,223-0,162+0,253) / 2=+0,004$
De acordo com os valores obtidos verifica-se que a velocidade de fotodegradação aumenta 0,088 unidades quando se passa de $\mathrm{pH} 5,5$ a $\mathrm{pH} 7,25$. Quando se acrescenta $\mathrm{AH}$, a constante de velocidade aumenta 0,026 unidades. Assim, pode-se dizer que o efeito do $\mathrm{pH}$ foi mais significativo do que a presença de $\mathrm{AH}$. Além disso, o efeito combinado das variáveis $\mathrm{pH}$ e AH é praticamente negligenciável (0,004 unidades), indicando que não existe correlação entre as variáveis.

A degradação fotoquímica do paration em água desionizada na presença de AH foi estudada utilizando-se uma lâmpada de mercúrio de média pressão com potência de 450 watts e comprimento de 11" 22. Os resultados mostraram uma degradação quase total do paration após 120 min de irradiação. No presente estudo, empregando-se uma lâmpada de mesma potência, porém, de menor comprimento (5"), observou-se uma degradação total do paration em menos de $30 \mathrm{~min}$. Essa diferença encontrada em ambos os experimentos pode ser atribuída à diferença de intensidade de luz emitida por ambas as lâmpadas, visto que a lâmpada de 5" apresenta uma intensidade de luz de $1,77 \times 10^{-9}$ einstein/s enquanto que a outra lâmpada (11") apresenta uma intensidade de luz de 7,41 x $10^{-10}$ einstein/s. As intensidades de luz foram determinadas através da actinometria com ferrioxalato de potássio ${ }^{23}$. Assim, verifica-se que a intensidade de luz influencia a velocidade de fotodegradação do paration.

Em adição, considerando-se que o $\mathrm{pH}$ foi mais eficiente que os AH na fotodegradação, outros experimentos foram realizados variando-se agora o comprimento de onda, mantendo-se o $\mathrm{pH} 7,25$, na ausência de AH.

A Figura 5 ilustra o comportamento fotoquímico do paration frente à radiação UV em $\lambda>313 \mathrm{~nm}$, na ausência de AH. Para facilitar a compreensão, foi adicionada à figura 5 o comportamento fotoquímico do paration quando da irradiação em $\lambda>280 \mathrm{~nm}$, na ausência de $\mathrm{AH}$ e pH 7,25.

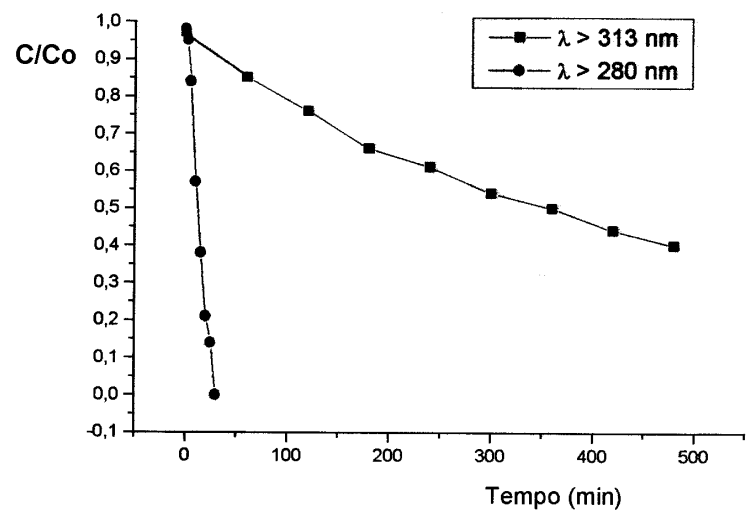

Figura 5. Concentração relativa de paration em água desionizada, na ausência de AH, pH 7,25, com irradiação em $\lambda>313 \mathrm{~nm}$ e $\lambda>280 \mathrm{~nm}$

A degradação do paration para este sistema mostra a influência do comprimento de onda empregado em estudos fotoquímicos. Como pode ser verificada, a degradação é mais lenta quando comparada aos sistemas anteriores. A constante de velocidade de fotodegradação para este sistema foi determinada em termos do logaritmo da concentração de paration remanescente em função do tempo. De acordo com os cálculos, o valor da constante de velocidade de fotodegradação foi $0,0018 \mathrm{~min}^{-1}$, valor este extremamente inferior aos dos sistemas anteriores. Neste caso, 6 horas de irradiação foi o tempo necessário para degradar $50 \%$ do paration, enquanto que em $\lambda>280 \mathrm{~nm}$ a degradação total ocorreu em menos de $30 \mathrm{~min}$. Vale lembrar que mesmo não havendo um efeito sensibilizador pelo $\mathrm{AH} \mathrm{em} \lambda>280 \mathrm{~nm}$, não implica que este comportamento seja o mesmo para comprimentos de onda diferentes. 
Embora as condições naturais sejam diferentes das de laboratório, o estudo fotoquímico com lâmpadas artificiais pode auxiliar na compreensão dos fotoprodutos formados, ou seja, qual ou quais possíveis fotoprodutos serão formados após a introdução de um pesticida no ambiente. Além disso, avaliar a importância da metodologia como uma alternativa para descontaminação de poluentes em águas.

\section{Influência do meio na degradação do paration em águas naturais}

A Figura 6 apresenta o comportamento fotoquímico do paration após irradiação UV em águas naturais em $\mathrm{pH}$ 5,5 na presença e ausência de AH.

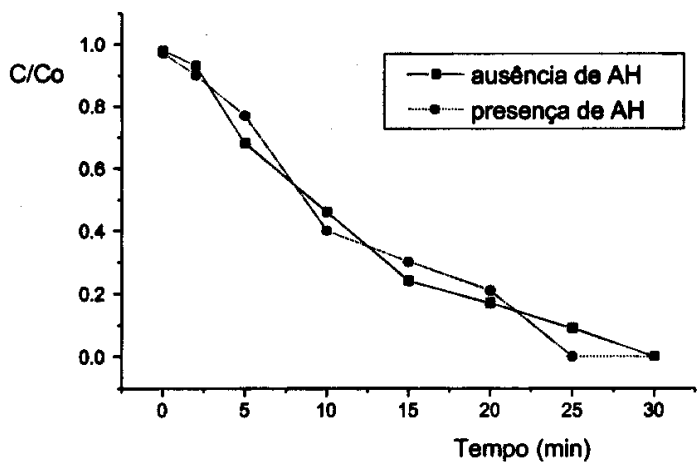

Figura 6. Concentração relativa de paration em águas naturais, na ausência e presença de $A H, p H 5,5$, com irradiação em $\lambda>280 \mathrm{~nm}$.

O paration também foi degradado em menos de 30 min para essa matriz, sendo 25 min e 30 min o tempo necessário para degradar totalmente o paration na presença e ausência de $\mathrm{AH}$, respectivamente. Porém, as constantes de velocidade de degradação do paration demonstraram uma menor velocidade de fotodegradação na presença e ausência de $\mathrm{AH}, \mathrm{k}=0,082 \mathrm{~min}^{-1}$ e k=0,089 $\mathrm{min}^{-1}$, respectivamente, quando comparadas aos valores obtidos em água desionizada, em mesmo $\mathrm{pH}$. Os valores das constantes cinéticas demonstram que os AH não aceleraram significativamente a degradação do paration. $\mathrm{O}$ mesmo comportamento foi observado para as amostras irradiadas em pH 7,25 na presença e ausência de AH, k=0,073 min $^{-1} \mathrm{e}$ $\mathrm{k}=0,081 \mathrm{~min}^{-1}$, respectivamente, Figura 7 .

Os resultados obtidos mostram, também, que o aumento do $\mathrm{pH}$ do meio não favoreceu a degradação.

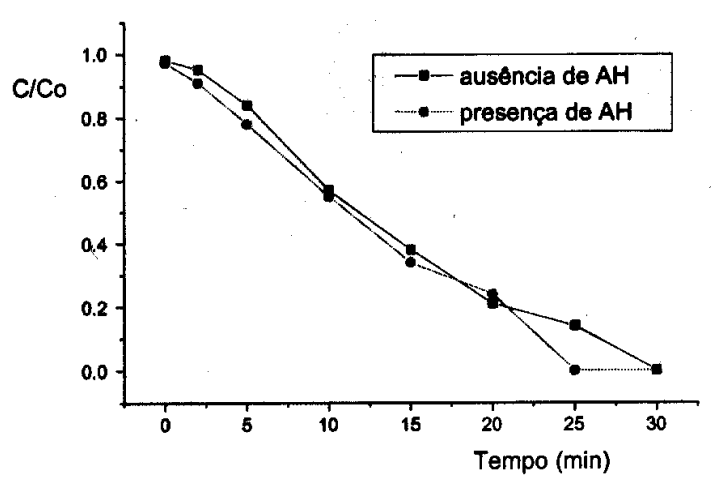

Figura 7. Concentração relativa de paration em águas naturais, na ausência e presença de $A H, p H 7,25$, com irradiação em $\lambda>280 \mathrm{~nm}$.
O planejamento fatorial ${ }^{21}$ também foi aplicado para este sistema para melhor compreensão dos resultados. A Tabela 3 apresenta o planejamento fatorial para calcular o efeito das variáveis $\mathrm{AH}$ e $\mathrm{pH}$ na degradação do paration.

Tabela 3. Resultados de um planejamento fatorial $2^{2}$ para estudar o efeito do $\mathrm{pH}$ e dos $\mathrm{AH}$ na fotodegradação

\begin{tabular}{|c|c|c|c|}
\hline \multicolumn{2}{|c|}{$\begin{array}{c}\text { Variável } \\
\mathrm{pH} \\
\mathrm{AH}\end{array}$} & \multirow{2}{*}{$\begin{array}{c}\text { Nível (-) } \\
5,5 \\
\text { Não } \\
\text { pH x AH }\end{array}$} & \multirow{2}{*}{$\begin{array}{c}\text { Nível (+) } \\
7,25 \\
\text { Sim } \\
\text { Resposta } \\
\left(\mathbf{k}, \text { min }^{-1}\right)\end{array}$} \\
\hline pH & $\mathbf{A H}$ & & \\
\hline- & - & + & 0,089 \\
\hline+ & - & - & 0,081 \\
\hline- & + & - & 0,082 \\
\hline+ & + & + & 0,073 \\
\hline
\end{tabular}

A partir dos valores da Tabela 3, o efeito dos $\mathrm{AH}$ e $\mathrm{pH}$ foi calculado como segue:

$$
\begin{aligned}
& \text { pH: }(-0,089+0,081-0,082+0,073) / 2=-0,008 \\
& \text { AH: }(-0,089-0,081+0,082+0,073) / 2=-0,007 \\
& \text { pH x AH: }(0,089-0,081-0,082+0,073) / 2=-0,0005
\end{aligned}
$$

De acordo com os valores obtidos verifica-se que a velocidade de fotodegradação diminui 0,008 unidades quando se passa de $\mathrm{pH}$ 5,5 a pH 7,25. Quando se acrescenta $\mathrm{AH}$, a constante de velocidade também diminuiu 0,007 unidades. Estes valores são praticamente iguais. Assim, pode-se dizer que o efeito do $\mathrm{pH}$ e AH não foi significativo na degradação fotoquímica para este sistema. Pode-se dizer, também, que o efeito combinado das variáveis $\mathrm{pH}$ e $\mathrm{AH}$ é praticamente negligenciável (0,0005 unidades), indicando que não existe correlação entre as variáveis.

A menor velocidade de fotodegradação do paration em águas naturais pode ser atribuída à complexidade da matriz, visto que existem outras moléculas no meio reacional, as quais podem competir com as moléculas de paration frente à radiação UV. A Tabela 4 resume os resultados da degradação fotoquímica do paration em função do meio.

Tabela 4. Resultados obtidos na degradação fotoquímica do paration em função do meio

\begin{tabular}{lccc}
\hline Matriz irradiada & $\mathrm{pH}$ & $\begin{array}{c}\text { Comprimento } \\
\text { de onda } \\
(\mathrm{nm})\end{array}$ & $\begin{array}{c}\text { Constante de } \\
\text { velocidade } \\
\left(\mathrm{k} \cdot \mathrm{min}^{-1}\right)\end{array}$ \\
\hline Água desionizada & 5,50 & $>280$ & $13,8 \times 10^{-2}$ \\
Água desionizada/AH & 5,50 & $>280$ & $16,1 \times 10^{-2}$ \\
Água desionizada & 7,25 & $>280$ & $22,3 \times 10^{-2}$ \\
Água desionizada/AH & 7,25 & $>280$ & $25,3 \times 10^{-2}$ \\
Água desionizada & 7,25 & $>313$ & $0,18 \times 10^{-2}$ \\
Águas naturais & 5,50 & $>280$ & $8,9 \times 10^{-2}$ \\
Águas naturais/AH & 5,50 & $>280$ & $8,2 \times 10^{-2}$ \\
Águas naturais & 7,25 & $>280$ & $7,3 \times 10^{-2}$ \\
Águas naturais/AH & 7,25 & $>280$ & $8,1 \times 10^{-2}$ \\
\hline
\end{tabular}

Deve ser salientado que as amostras irradiadas não sofreram nenhum tratamento prévio, como filtração, para retirar eventuais partículas em suspensão. Essas partículas podem atenuar a luz emitida e, 
assim, inibir a fotodegradação. A Figura 8 apresenta o espectro de absorção das águas utilizadas no experimento.

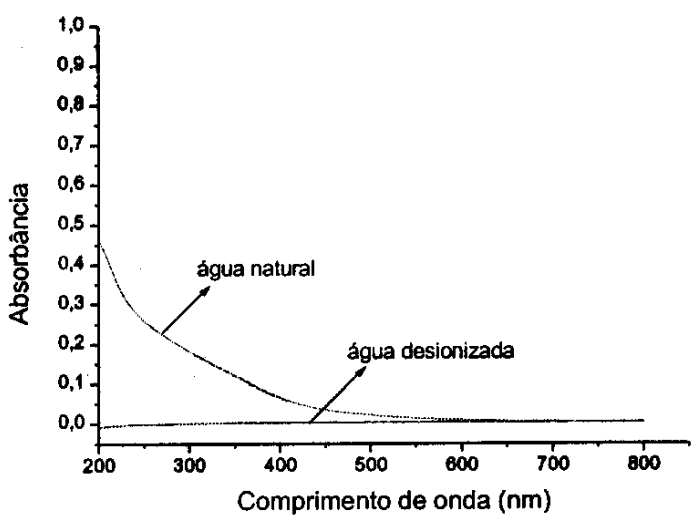

Figura 8. Espectro UV-Vis das amostras das águas utilizadas nos experimentos fotoquímicos.

A análise por HPLC-UV das amostras irradiadas na presença e ausência de $\mathrm{AH}$ mostrou a formação de alguns fotoprodutos intermediários no meio aquoso. A Figura 9 apresenta um cromatograma de uma das amostras irradiadas.

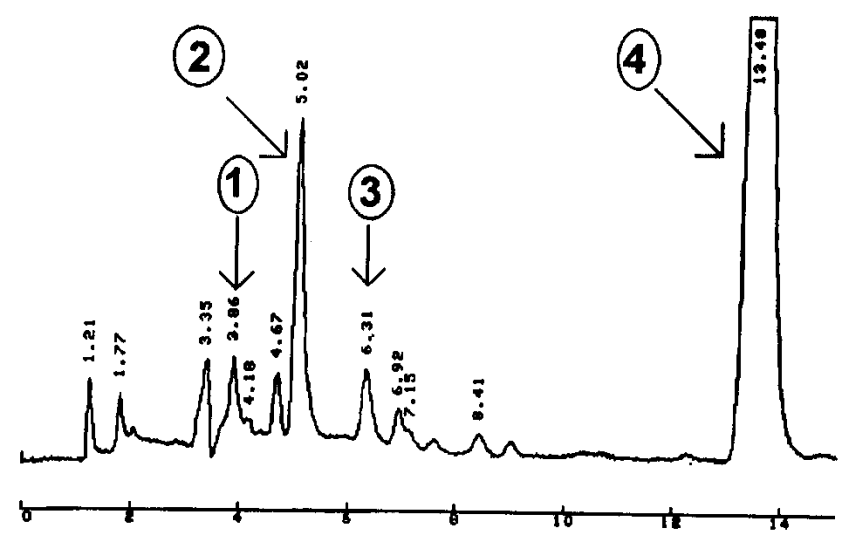

Figura 9. Cromatograma (HPLC-UV) do extrato em metanol de paration irradiado durante 10 minutos, na ausência de $A H, p H 7,25$, em $\lambda>280 \mathrm{~nm}$. Os números representam os fotoprodutos aminofenol (1), p-nitrofenol (2) $e$ paraoxon (3). O número 4 representa o paration.

Pela comparação dos tempos de retenção dos picos 1, 2 e 3 com os tempos de retenção de padrões autênticos, identificou-se o pico 1 como sendo o fotoproduto aminofenol, o pico 2 como p-nitrofenol e o 3 como paraoxon. A confirmação dos fotoprodutos foi feita por GC-MS por meio da comparação entre os espectros de massas dos compostos encontrados com padrões puros, estudo da fragmentação de espectro de massas dos compostos e comparação entre o espectro de massas do composto com a biblioteca de dados do GC-MS. Estes fotoprodutos também foram encontrados na degradação biológica do paration ${ }^{20}$.

Embora os processos de degradação fotoquímicos e biológicos sejam diferentes, os fotoprodutos paraoxon, aminofenol e p-nitrofenol são os mesmos. De acordo com Barik e Sethunathan ${ }^{20} \mathrm{e}$ Sethunathan et al. ${ }^{24}$, o paraoxon é resultado da oxidação do grupo fosforotioato. Já o p-nitrofenol e aminofenol são espécies resultantes da hidrólise do grupo fosfato.
A Tabela 5 apresenta a relação massa/carga elétrica dos principais fragmentos encontrados.

Tabela 5. Relação massa/carga elétrica e tempo de retenção dos principais fragmentos encontrados

\begin{tabular}{lcc}
\hline Composto & $\begin{array}{c}\text { Tempo } \\
\text { de retenção } \\
(\min )\end{array}$ & Massa/carga \\
\hline p-Aminofenol & 6,7 & $109,80,53$ \\
p-Nitrofenol & 10,5 & $139,123,109,98,81,65$ \\
Paraoxon & 15,2 & $275,247,139,109,81,65$ \\
Paration & 16,1 & $291,235,155,139,125,109,97,81,65$ \\
\hline
\end{tabular}

Para as amostras irradiadas em $\mathrm{pH}$ 5,5, o p-nitrofenol foi o principal fotoproduto formado, sendo que a maior porcentagem de formação ocorreu em 5 min de irradiação (8,0 e 5,4\% na presença e ausência de AH, respectivamente). Em pH 7,25 o p-nitrofenol também foi o fotoproduto majoritário, tendo sua maior porcentagem de formação após 10 min de irradiação $(9,0$ e 7,5\% na ausência e presença de AH, respectivamente).

O paraoxon foi formado em baixa concentração em pH 5,5. Em pH 7,25, foi determinado apenas em solução irradiada na ausência de AH. Isto sugere que o AH pode influenciar sua fotodegradação. Tal fato é de grande importância, visto que o paraoxon é mais tóxico que o paration.

O fotoproduto aminofenol foi formado em baixa concentração em ambas as soluções, ou seja, em pH 5,5 e 7,25 na presença e ausência de AH.

Em $\lambda>313 \mathrm{~nm}$ foi verificada apenas a presença p-nitrofenol na concentração máxima de formação de $5,8 \%$. No entanto, outros compostos não extraídos com acetato de etila podem estar sendo formados. Ao que parece, a formação de fotoprodutos depende do comprimento de onda emitido. Este mesmo comportamento foi constatado em outro trabalho ${ }^{25}$. Os autores verificaram que a fotodegradação do fenuron levou à formação de diferentes fotoprodutos quando da irradiação em comprimentos de onda de $254 \mathrm{~nm}$ e $365 \mathrm{~nm}$.

Os resultados demonstraram que os fotoprodutos também sofreram degradação em função do tempo de irradiação.

\section{CONCLUSÕES}

De acordo com as condições experimentais avaliadas neste estudo, os resultados indicaram que a degradação do paration ocorreu somente através da degradação fotoquímica e não através de hidrólise ou degradação biológica. Em $\lambda>280 \mathrm{~nm}$ a degradação do paration foi mais significativa em água desionizada e em meio alcalino do que quando comparada à degradação em águas naturais, nas mesmas condições.

A velocidade de fotodegradação dependeu do comprimento de onda aplicado. Em $\lambda>313 \mathrm{~nm}$ a degradação fotoquímica do paration foi extremamente lenta quando comparada com os valores obtidos em $\lambda>280 \mathrm{~nm}$. Além disso, foi verificado que a formação de fotoprodutos pode depender do comprimento de onda.

A porcentagem de fotoprodutos formados foi muito pequena. Tal fato é um indicativo da possível destruição deles com a radiação UV.

A radiação UV pode ser uma alternativa de descontaminação do paration em matrizes aquosas.

\section{AGRADECIMENTOS}

Os autores agradecem à FAPESP, CNPq e CAPES, pelo apoio financeiro. 


\section{REFERÊNCIAS}

1. Bachman, J.; Patterson, H.; Environ. Sci. Technol. 1999, 33, 874.

2. Ruppert, G.; Bauer, R.; J. Photochem. Photobiol. A 1993, 73, 75

3. Comninellis, C. In Environment oriented electrochemistry; Sequeira, C.A.A., Ed.; Elsevier; Amsterdam, 1994; p.77.

4. Photocatalytical purification and a treatment of water and air; Ollis, D.F.; Ekabi, H.A., Ed.; Elsevier; Amsterdan, 1993.

5. Bahnemann, D.; Cunningham, J.; Fox, M.A.; Pellizetti, E.; Pichat, P.; Serpone, N. In Aquatic and surface photochemistry; Helz, G.R.; Zepp, R.G.; Crosby, D.G., Ed.; Lewis Publishers; Boca Raton, 1994; p 261.

6. Tahiri, H; Chouy, Y.A.I.; Herrmann, J.M.; J. Photochem. Photobiol. A 1998 , $114,219$.

7. Legrini, O.; Oliveros, E.; Braun, A.M.; Chem. Rev. 1993, 93, 671.

8. Mathew, R.; Khan, S.U.; J. Agric. Food Chem. 1996, 44, 3996.

9. Baster, R.M.; Carey, J.H.; Nature 1983, 306, 575.

10. Zepp, R.G.;Braun, A.M.; Hoigné, J.; Leenherr, J.A.; Environ. Sci. Technol. 1987, 21,485 .

11. Haag, W.H.; Hoigné, J.; Environ. Sci. Technol. 1986, 20, 341.

12. Aguer, J.P.; Richard, C.; J. Photochem. Photobiol. A 1996, 93, 193.

13. Frimmel, F.H.; Hessler, D.P. In Aquatic and surface photochemistry; Helz,
G.R.; Zepp, R.G.; Crosby, D.G., Ed.; Lewis Publishers; Boca Raton, 1994; p.137.

14. Zepp, R.G.; Schlotzhauer, P.F.; Sink, R.M.; Environ. Sci. Technol. 1985, 19,74 .

15. Nogueira, R.F.P.; Jardim, W.F.; Sol. Energy 1996, 56, 471.

16. Nogueira, R.F.P.; Jardim, W.F.; Quim. Nova 1998, 21, 69.

17. Kamiya, M.; Kameyama, K.; Chemosphere 1998, 36, 2227.

18. Stevenson, F.J.; Humus Chemistry, Genesis, Composition, Reactions; John Wiley \& Sons; New York, 1982.

19. Landgraf, M.D; Silva, S.C., Rezende, M.O.O.; Anal. Chim. Acta 1997, 368, 155.

20. Barik, S.; Sethunathan, N.; J. Environ. Qual. 1978, 7, 346.

21. Neto, B.B.; Scarminio, I.S.; Bruns, R.E.; Planejamento e otimização de experimentos; Editora da Unicamp; Campinas, 1995; p.61.

22. Santos, F.F.; Dissertação de Mestrado; Instituto de Química de São Carlos; USP, São Carlos, 1996.

23. Rabek, J.P.; Experimental methods in photochemistry and photophysics; John Wiley \& Sons; New York, 1982; p.944.

24. Sethunathan, N.; Siddaramappa, R.; Rajaram, K.P.; Barik, S.; Wahid, P.A.; Res. Rev. 1977, 68, 91.

25. Aguer, J.P.; Richard, C.; Pestic. Sci. 1996, 46, 151 